\title{
Nine Ball Pool Handicap System: Estimating the APA Method
}

\author{
Salvador Lopez \\ Associate Professor of Economics \\ 1601 Maple St \\ Department of Economics \\ University of West Georgia \\ Carrollton, GA 30118, USA
}

\begin{abstract}
Most amateur pool players who want to play and compete with other players join the American Pool players Association (APA) and participate in a league at a local bar or location with APA affiliation. APA assigns skill levels using a handicap system that is not available to the public. Specifically, in the game of nine ball, each player is assigned a skill level based on a secret formula designed by APA. Given the limited information provided by the score sheet that serves as input to determine such level, this paper attempts to estimate the method or at least develop one that is consistent with both the points system used and sample data results.
\end{abstract}

Keywords: handicap, rating system, competitive balance, single equation model, amateur sports.

JEL classification: $\mathrm{C} 2, \mathrm{Z} 2$

\section{Introduction}

In different sports, a handicap system is a method of offsetting the different abilities of competitors, so their chances of winning are equalized. Handicap systems are known to be used in horse racing, golf, bowling, tennis table, and other sports. For example, Bingham (2000) and Swartz (2009)analyze past and present systems in golf. Chan and Singal (2016)(2018) develop a Markov Decision Process-based (MDP) method and apply a Bayesian regression model to design a handicap system in tennis. Johnson (2012) and Manzione (2018) criticize the six and eight-pin handicap system in bowling. Champagne, Coupet and Gerville (2013) develop a handicap system for table tennis using a simple probabilistic method. In pool, however, very little has been written about the handicap system used in nine-ball game. In the literature, I found only one related paper, written by O'Boyle (2014), which discusses techniques of neutralization and cheating in both eight ball and nine ball, but nothing on the handicap system itself.

\section{Method}

APA does not reveal the method used to assign skill levels to players. In fact, in their website, this is what is said about the calculation of skill levels: "Skill levels are maintained, calculated and updated by the Local League Office. The process includes a number of factors such as the application of specific mathematical formulas to the data on the weekly score sheets, win/loss records, Higher Level Tournament performance, qualitative judgment by Handicap Advisory Committees, and other considerations. You are asked to refrain from attempting to keep your own records as it is a generally disruptive practice. The APA appreciates your cooperation with this policy." In other words, their handicap system is property of APA and it is not available to the public. Based on such claim and the limited information known to the public, I have made an estimation of the APA method to assign skill levels in the game of nine ball. First, the known information is that there are nine skill levels, from one to nine. There are also nine required or goal points for each skill level ranging from 14 for skill level one to 75 for skill level nine. Table 1 below summarizes the skill levels and their corresponding points required to win a match.

\footnotetext{
${ }^{1}$ Found at their website: https://poolplayers.com/faq-handicaps-calculated/
} 
Table 1: skill levels and points to win

\begin{tabular}{|l|l|}
\hline Skill Level & Points required to win \\
\hline 1 & 14 \\
\hline 2 & 19 \\
\hline 3 & 25 \\
\hline 4 & 31 \\
\hline 5 & 38 \\
\hline 6 & 46 \\
\hline 7 & 55 \\
\hline 8 & 65 \\
\hline 9 & 75 \\
\hline
\end{tabular}

Second, when a match between two players starts, they play as many games as necessary to reach the number of points required. Each ball counts for one point except the nine ball, which counts for two points. So, at the end of one game, there are ten points that can be earned by both players. Two independent players from both teams keep the score of the match. After the two players complete their respective turns to shoot, one inning is scored. After the players complete all the games necessary for one of them to reach his/her goal number of points, the match ends and the winner is awarded a number of "match points" while the loser also earns a number of points, which are less than the winner's. The match points awarded to both players total 20 points assigned as $20-0,19-1,18-2$, and so on until a last score of 12-8 is awarded, depending on the number of points scored by the loser of the match. Table 2 below shows all possible points awarded.

Table 2: points awarded at the end of every match

\begin{tabular}{|l|l|l|l|l|l|l|l|l|l|}
\hline $\begin{array}{l}\text { Loser's } \\
\text { SL }\end{array}$ & $\mathbf{2 0 - 0}$ & $\mathbf{1 9 - 1}$ & $\mathbf{1 8 - 2}$ & $\begin{array}{l}\text { SCORE } \\
\mathbf{1 7 - 3}\end{array}$ & $\begin{array}{l}\text { OF } \\
\mathbf{1 6 - 4}\end{array}$ & $\begin{array}{l}\text { MATCH } \\
\mathbf{1 5 - 5}\end{array}$ & $\mathbf{1 4 - 6}$ & $\mathbf{1 3 - 7}$ & $\mathbf{1 2 - 8}$ \\
\hline 1 & $<3$ & 3 & 4 & $5 \& 6$ & 7 & 8 & $9 \& 10$ & 11 & $12 \& 13$ \\
\hline 2 & $<4$ & $4 \& 5$ & $6 \& 7$ & 8 & $9 \& 10$ & $11 \& 12$ & $13 \& 14$ & $15 \& 16$ & $17 \& 18$ \\
\hline 3 & $<5$ & $5 \& 6$ & $7-9$ & $10 \& 11$ & $12-14$ & $15 \& 16$ & $17-19$ & $20 \& 21$ & $22-24$ \\
\hline 4 & $<6$ & $6-8$ & $9-11$ & $12-14$ & $15-18$ & $19-21$ & $22-24$ & $25-27$ & $28-30$ \\
\hline 5 & $<7$ & $7-10$ & $11-14$ & $15-18$ & $19-22$ & $23-26$ & $27-29$ & $30-33$ & $34-37$ \\
\hline 6 & $<9$ & $9-12$ & $13-17$ & $18-22$ & $23-27$ & $28-31$ & $32-36$ & $37-40$ & $41-45$ \\
\hline 7 & $<11$ & $11-15$ & $16-21$ & $22-26$ & $27-32$ & $33-37$ & $38-43$ & $44-49$ & $50-54$ \\
\hline 8 & $<14$ & $14-19$ & $20-26$ & $27-32$ & $33-39$ & $40-45$ & $46-52$ & $53-58$ & $59-64$ \\
\hline 9 & $<18$ & $18-24$ & $25-31$ & $32-38$ & $39-46$ & $47-53$ & $54-60$ & $61-67$ & $68-74$ \\
\hline
\end{tabular}

Based on this limited information, my personal experience as a player who moved from level 4 to level 7 in a period of one year, and the experience described by other players, I conclude that the only handicap system that can be used should be based on the goal points assigned to all players and the "speeds" that different players have. Such speed is measured by the points per inning scored by each player after a match is completed. Better players, obviously, will record higher points per inning and lesser players lower points. Using basic algebra, lets define points required to win as $P_{i}$ and the points per inning as $p_{i}$. A basic and logical proposal is that the ratio of total points must be equal to the ratio of speeds, so the chances to win for every player would be the same. For example, if two car drivers can drive at speeds of $180 \mathrm{mph}$ and $60 \mathrm{mph}$ respectively, in a race of 30 minutes, driver one must drive a distance of 90 miles while driver two must drive 30 miles. In other words, the ratio of the distance, or 90 miles $/ 30$ miles $=3$, must match the ratio of the speeds: 180 miles/hour divided by 60 miles per hour $=3$ too. Based on that idea, I conclude that a fair handicap system must guarantee that each player will take the same amount of innings to complete their own points to win, the same way the two drivers complete their distances in half-an-hour each. In other words, the ratio of total points required by a skill level 9 to a skill level 1 must match the ratio of their corresponding points per inning ("speed"), or, P9/P1 $=$ p9/p1. Since 75/15 equals 5 , the ratio of speeds must match that ratio too. ${ }^{2}$ Now, as far as I know, it is impossible to develop a mathematical model that could lead to the determination of those two expected points per inning (speeds). However, from personal experience, I estimated a simple ratio of 5 to 1 as appropriate. I used these numbers based on my own experience as a player and observant of

${ }^{2}$ To facilitate calculation, total points to win for skill level one is assumed to be 15 instead of 14 . Later, calculations are done using 14 , as it should be. 
the game. I was one of the few players who always paid close attention to the points per inning. As I was moving from a skill level 4 to a skill level 7, my average points per inning improved from about 3 to 4 or slightly higher (but not 5). Since $\mathrm{P} 9 / \mathrm{P} 1=\mathrm{p} 9 / \mathrm{p} 1$, cross-multiplying leads to $\mathrm{P} 9 / \mathrm{p} 9=\mathrm{P} 1 / \mathrm{p} 1$, but those ratios are actually the expected number of innings to win. Whenever a player wins a match, their speed can be calculated by dividing the points earned by the number of innings played, or $\mathrm{Pi} / \mathrm{I}$, where $\mathrm{I}=$ amount of innings played. In other words, $\mathrm{pi}=\mathrm{Pi} / \mathrm{I}$, or $\mathrm{Pi} / \mathrm{pi}=\mathrm{I}$. Since P9/p9 = 75/5 = 15, the same must apply to skill level one: P1/p1 = 15, or 15/1 = 15 .

Using the proposed method of fifteen expected innings to reach the required number of points across all skill levels, expected points per inning can be calculated for every category. Table 3 below shows the results:

Table 3: expected points per inning given expected 15 innings to win a match

\begin{tabular}{|l|l|}
\hline Skill Level & Expected points per inning \\
\hline 1 & $14 / 15=0.933$ \\
\hline 2 & $19 / 15=1.266$ \\
\hline 3 & $25 / 15=1.666$ \\
\hline 4 & $31 / 15=2.066$ \\
\hline 5 & $38 / 15=2.533$ \\
\hline 6 & $46 / 15=3.066$ \\
\hline 7 & $55 / 15=3.666$ \\
\hline 8 & $65 / 15=4.333$ \\
\hline 9 & $75 / 15=5.000$ \\
\hline
\end{tabular}

Given that the numbers shown in table 2 are expected or average values, within each skill level, a normal distribution is assumed with few weak players and a few strong players. For example, for skill levels 4, figure 1 below shows such distribution.

Figure 1: Normal distribution at expected $\mathrm{pi}=\mathbf{2 . 0 6 6}$ for $\mathrm{SL}=4$.

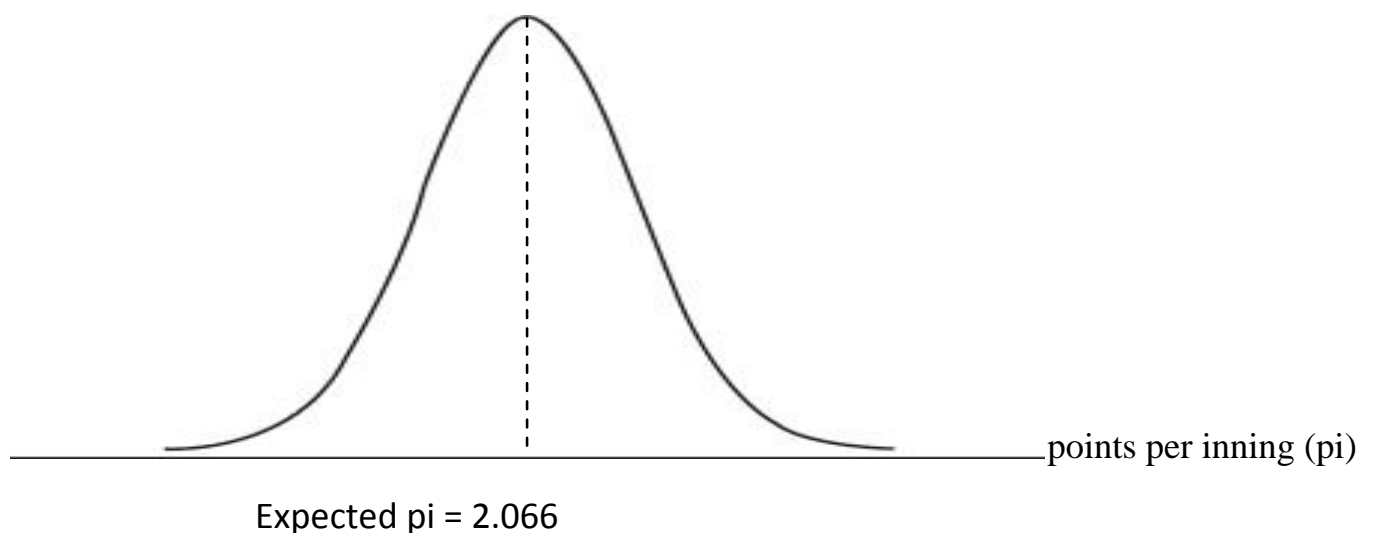

Given a continuous number of points per inning across all skill levels, or continuous tails of normal distributions, I estimated minimum, expected and maximum points per inning for every skill level. For example, for skill levels 4 and 5, the expected pis are 2.066 and 2.533 respectively. The average of those two values is $(2.066+2.533) / 2=2.299$. That would be the end of the upper tail for skill level 4 and beginning of lower tail for skill level 5 . In other words, the maximum speed for skill level 4 and the minimum speed required for skill level 5. Table 4 below shows the rest of values. 
Table 4: minimum, expected and maximum pi's per skill level

\begin{tabular}{|l|l|l|l|}
\hline Skill Level & Minimum pi & Expected pi & Maximum pi \\
\hline 1 & 0.766 or lower & 0.933 & 1.09 \\
\hline 2 & 1.10 & 1.266 & 1.465 \\
\hline 3 & 1.466 & 1.666 & 1.865 \\
\hline 4 & 1.866 & 2.066 & 2.299 \\
\hline 5 & 2.300 & 2.533 & 2.799 \\
\hline 6 & 2.800 & 3.066 & 3.365 \\
\hline 7 & 3.366 & 3.666 & 3.999 \\
\hline 8 & 4.000 & 4.333 & 4.665 \\
\hline 9 & 4.666 & 5.000 & 5.333 or higher \\
\hline
\end{tabular}

\section{Data}

I live in a city where a small group of pool players and entrepreneurs decided to open a billiards' club, but they did not want to affiliate to APA. They decided, however, to continue using a similar score sheet to record wins and losses. After the first season, I was given access to the data from a total of 14 teams and 107 players who played during a period of 14 weeks. On average, players played about 7-8 matches. A total of 52 played for APA before and, with a few exceptions, their skill levels continued at the same level. Every Tuesday night, two teams played 5 matches for a total projected 980 matches. However, some of those matches were not played due to shortage of players. Teams one through eight were mostly composed of old players with previously known skill levels, while teams nine through fourteen consisted of new teams and a special committee of experienced players assigned skill levels of four to new male players and three to new female players, which at the time was consistent with APA method. Starting in 2019, APA assigns skill level three to all beginners regardless of gender.

\section{Findings}

By the end of the season, teams one through eight, mainly composed of more experienced players won 57 percent of their matches while teams nine through fourteen ended up with a win percentage of 39. Such result was mainly due to a high number of inexperienced players joining new teams. They had assigned skill levels four or three but most of them were level two or one. The committee slowly adjusted their skill levels, but it occurred only until these players had accumulated several losses. The committee leaders adopted a "20-inning" method instead of 15 since they were convinced that such number was the one APA used. They claimed to have special information from somebody who used to work for APA, but no mathematical justification was provided. For that reason, I made an empirical evaluation of both methods. I used the data for the 52 already-established players and made a prediction of their skill levels. I took their last known skill levels and subtracted the predicted skill levels, so negative values would indicate an underestimation of skill levels while positive values would be an overestimation of skill levels. Then, I added all the differences. The results are summarized in table 5 below:

Table 5: estimation of previous skill levels of 52 players using 15 and 20inning methods

\begin{tabular}{|l|l|l|}
\hline Method & $\begin{array}{l}\text { Under/Over estimation of skill } \\
\text { levels (total points) }\end{array}$ & $\begin{array}{l}\text { Average under/over estimation of } \\
\text { skill levels }\end{array}$ \\
\hline 20 innings & +30 & +0.58 \\
\hline 15 innings & -15 & -0.29 \\
\hline
\end{tabular}

As may be noticed, a perfect prediction would have resulted in zero under or over estimation of skill levels. However, that is practically impossible since first, the data were limited to about 7-8 matches per player. Second, players do not always perform at their skill levels. Third, the tables being used were brand new, which tends to affect player performance. Fourth, some of the players had not played in a while (at least one year). On the other hand, clearly, the 20-inning method tends to over predict skill levels while the 15-inning method tends to under predict them. Finally, table 4 clearly shows that the 15 -inning method is a better predictor of skill levels. In fact, it is about twice more accurate than the 20-inning method.

The main objective of this paper is to estimate the average number of innings played by those players who won their matches and compare it to the expected fifteen innings suggested in the method section earlier in the paper. Since the points per inning times number of innings played equal total points scored ( $\mathrm{pi}$ x I $=\mathrm{Pi}$ ), I ran a regression between pi and $\mathrm{Pi}$ to estimate such equation setting the vertical intercept at zero. 
It is important at this point to clarify why I did not just simply calculated the average innings played, or took the average points scored divided by the average points per inning. First, a simple average, or "naïve" method, is a positively-biased estimate since it is influenced by unusually large number of innings used by some players to complete their matches. That average was 19.45 innings. The second estimate, or "improved" method, is 17.33 innings. Figure 2 below shows why this is not a very good estimate either. The graph is from hypothetical data of five observations of variables $\mathrm{X}$ and $\mathrm{Y}$ (equivalent to pi and $\mathrm{Pi}$ ). The line $\mathrm{y}=17.82 \mathrm{X}$ is a line that goes through the average of $\mathrm{X}$ and $\mathrm{Y}$, as suggested by such method. As you may notice, the slope of that line, 17.82, is not a best-fit line since it is forced to pass through that point and not through the middle of all points, as it is done by the Ordinary Least Squares (OLS) method, resulting in a higher estimate of the slope (or "average/expected innings").

\section{Figure 2: difference between estimating average innings using the ratio of averages versus a regression} coefficient from the OLS method using hypothetical data for two variables $\mathrm{X}$ and $\mathrm{Y}$.

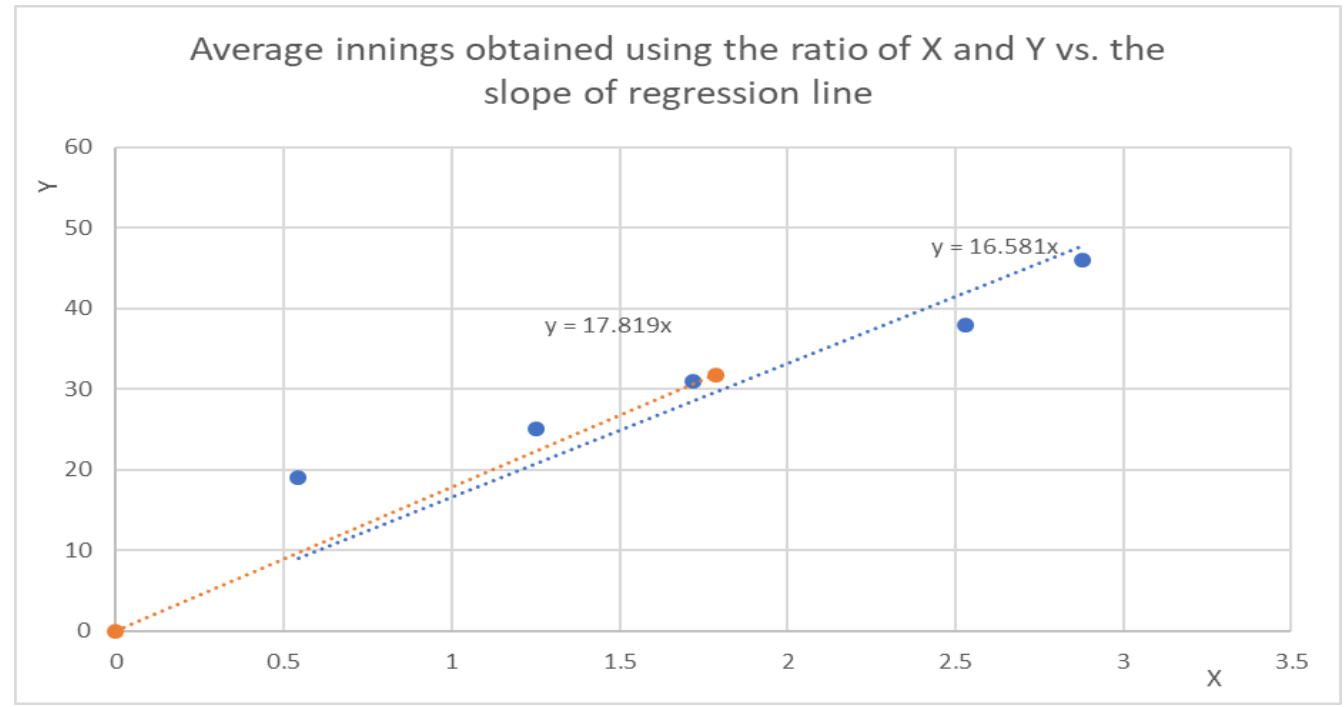

Using the actual data from the sample, the regression equation obtained was $\mathrm{Pi}=15.904 \mathrm{x}$ pi with a $\mathrm{p}$-value equal to zero. Such result confirms that the 15 -inning method is accurate and appropriate. Figure 3 below shows the equation line using the actual sample data.

Figure 3: OLS equation using data for all players who won their matches

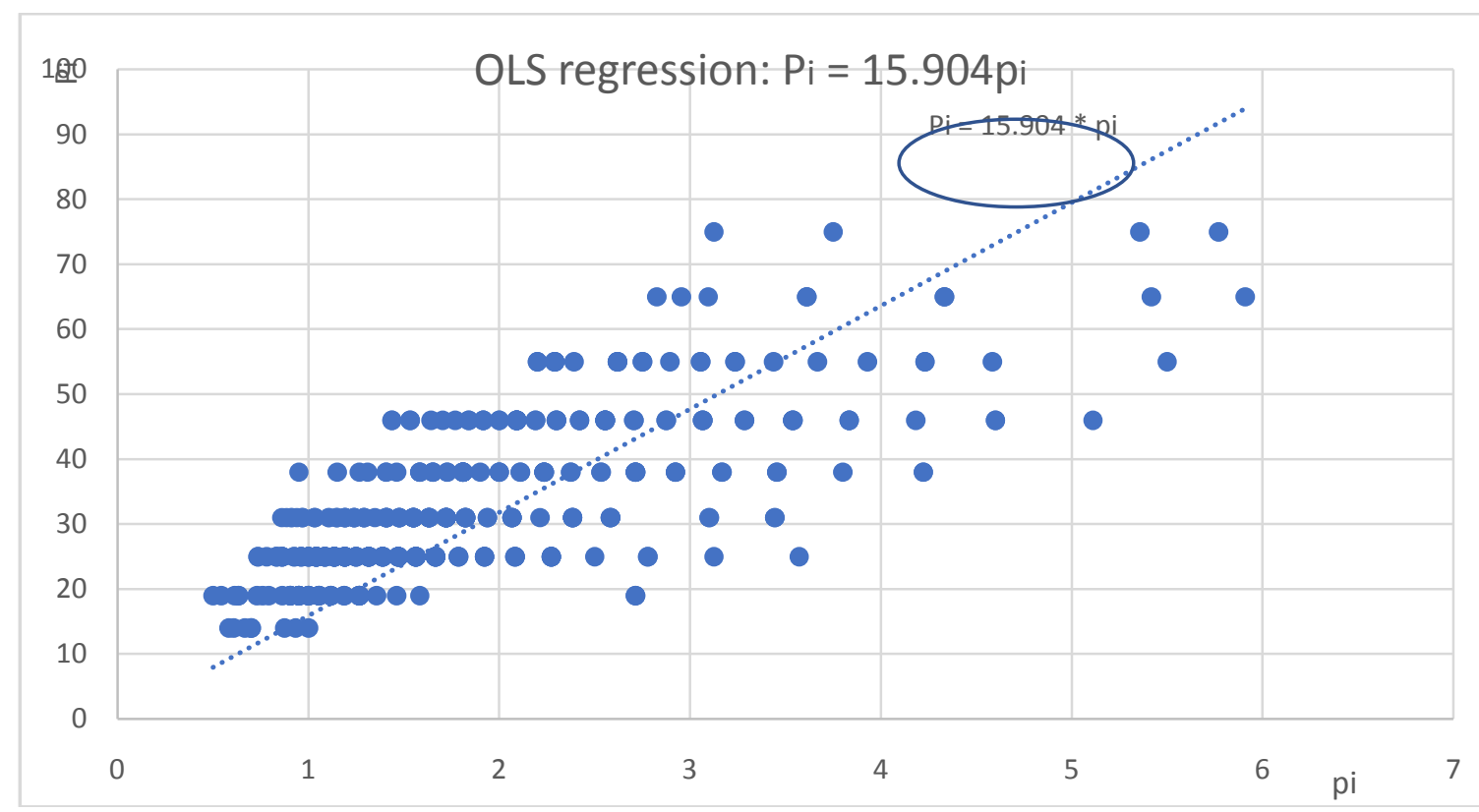




\section{Conclusion and recommendations}

My main conclusion is that the assumption of a handicap system that uses 15 expected innings to win a match seems to be very reasonable and accurate. The data from the sample used confirms it by running a linear regression between points scored and points per inning for 107 players who played about 7-8 matches during a period of 14 weeks. However, players, especially the new ones, were assigned higher levels, which led to a disproportionate number of wins or competitive imbalance. The same imbalance was observed between teams that had experienced players, or players with records, and the teams without experienced and established players. Obviously, consistency of the results needs to be further tested by using a bigger sample. That can be done after the second season ends. Another recommendation for future research is the use of simulation to obtain wins and losses for players who would have received an updated skill levels from the very start of the season. My expectation with such simulation is that competitive balance would be improved significantly leading closer to an expected fifty-win percentage for all players.

\section{References}

Bingham, D. \& Swartz, T. (2000). Equitable Handicapping in Golf. The American Statistician, 54(3), 170-177.

Champagne, L., Coupet, A., \& Gerville-Reache, L. (2013). Elaboraring a Rule for Game Handicap in Table Tennis: A Probabilistic Approach. 13th ITTF Sports Science Congress, May 11-12, pp. 70-71. Paris, France.

Chan, T. C. (2016). A Markow Decision Process-Based Handicap System for Tennis. Journal of Quantitative Analysis in Sports, 12, 179-188.

Chan, T., \& Singal, R. (2018). A Bayesian Regression Approach to Handicapping Tennis Players Bases On A Rating System. Journal of Quantitative Analysis in Sports, 14(3), 131-141.

Johnson, B. (2012, March). Opponents' Handicap is Not Enough ... This Time. Bowlers Journal International, 27.

Manzione, G. (2018, May). Mutiny in Munich. Bowlers Journal International, 21.

O'Boyle, T. (2014, February). Techniques of Neutralization and Cheating in a National Pool League. International Journal of Business and Social Science, 5(2), 58-65.

Swartz, T. (2009). A New Handicapping System for Golf. Journal of Quantitative Analysis in Sports, 5(2), 9. 